\title{
Responses to persuasive messages encouraging professional help seeking for depression: comparison between individuals with and without psychological distress
}

\author{
Machi Suka*, Takashi Yamauchi and Hiroyuki Yanagisawa
}

\begin{abstract}
Background: The persuasive effect of health messages can depend on message features, audience characteristics, and target behaviors. The objective of this study was to compare the responses to persuasive messages encouraging professional help seeking for depression between individuals with and without psychological distress.

Methods: A cross-sectional web-based survey was conducted on Japanese adults aged 35-45 years, who randomly received one of three persuasive messages that aimed to promote help-seeking intentions for depression. The primary message statements were as follows: (1) depression can happen to anyone, (2) depression needs treatment, and (3) depression improves with treatment. Participants rated the messages in terms of comprehensibility, persuasiveness, emotional response, and intended future use. Help-seeking intention for depression was measured using vignette methodology before and after exposure to the messages. Eligible participants who had not received medical treatment for their mental disorders were classified as either distressed (K6 score $\geq 5, N=824$ ) or nondistressed (K6 score < 5, N=1133) and analyzed.

Results: No significant differences in comprehensibility or persuasiveness scores were observed between the messages, but the distressed group had significantly lower scores than the non-distressed group. Negative emotional responses such as surprise, anger, fear, sadness, guilt, and anxiety were significantly stronger when reading message 2 , while a positive emotional response such as happiness was significantly stronger when reading message 3. These emotional responses were more prominent in the distressed than in the non-distressed group. After reading messages 1, 2, and 3, the proportions of participants in the distressed group who reported having a positive help-seeking intention increased by $35.1 \%, 32.1 \%$, and $27.7 \%$, respectively, and by $6.4 \%, 17.3 \%$, and $15.2 \%$, respectively in the non-distressed group. Multiple logistic regression analysis among participants having no helpseeking intention before exposure to the messages showed that message 2 had a significantly greater effect of increasing help-seeking intentions in the non-distressed group.

(Continued on next page)
\end{abstract}

\footnotetext{
* Correspondence: suka@jikei.ac.jp

Department of Public Health and Environmental Medicine, The Jikei

University School of Medicine, 3-25-8 Nishi-Shimbashi, Minato-ku, Tokyo

105-8461, Japan
}

(c) The Author(s). 2019 Open Access This article is distributed under the terms of the Creative Commons Attribution 4.0 International License (http//creativecommons.org/licenses/by/40/) which permits unrestricted use, distribution, and reproduction in any medium, provided you give appropriate credit to the original author(s) and the source, provide a link to the Creative Commons license, and indicate if changes were made. The Creative Commons Public Domain Dedication waiver (http://creativecommons.org/publicdomain/zero/1.0/) applies to the data made available in this article, unless otherwise stated. 
(Continued from previous page)

Conclusion: The exposure to persuasive messages may promote help-seeking intentions for depression. It seems

likely that loss framing will work better than neutral and gain framing. Meanwhile, the responses to persuasive

messages may differ to some extent between distressed and non-distressed individuals, as individuals with

psychological distress are likely to be more susceptible to persuasive messages than those without.

Trial registration: Not applicable; this is not a report of intervention trial.

Keywords: Depression, Help-seeking, Persuasive message, Questionnaire survey, Japan,

\section{Background}

Depression can have a profound impact on daily functioning and quality of life. Early detection and appropriate treatment can promote remission, prevent relapse, and reduce the burden of the disease [1]. However, many affected individuals delay in seeking professional help and fail to receive effective treatment $[2,3]$. The results of numerous studies performed to examine the effectiveness of interventions designed to promote help-seeking for depression have suggested that improvements were achievable in some aspects of help-seeking, but the effect sizes were small [4]. Thus, additional studies are needed to identify more effective approaches for improving public attitudes, intentions, and behaviors.

The study and use of communication strategies to inform and influence individual and community decisions that enhance health is referred to as health communication [5]. Communicating persuasive messages may be able to make significant contributions to solve the problem of untreated depression, but their effects would depend on the message features, audience characteristics, and target behaviors [6-8]. Previous studies suggested a potential negative effect of depression help-seeking messages (i.e., boomerang effect) $[9,10]$. For more effective messaging, providers need to incorporate audience perspective to message design $[11,12]$. Pretesting messages with audience members is recommended for this purpose [5].

We launched a research project to develop effective health communication interventions for encouraging help-seeking in people at risk of suicide. First, we developed rating scales to measure audience perceptions regarding the effectiveness of health messages among Japanese individuals [13]. Second, we intended to develop effective public health messages that would promote help-seeking intentions for depression [14]. From a public health perspective, increasing public knowledge about depression is essential to improve help-seeking for depression. Thus, our target audience was the general public, excluding individuals who had received medical treatment for their mental disorders. Since the audience can be either distressed or non-distressed, we needed to know whether the effectiveness of depression help-seeking messages is influenced by audience's psychological distress level. To achieve this objective, we compared the responses to persuasive messages encouraging professional help seeking for depression between individuals with and without psychological distress.

\section{Methods}

The Step approach to Message Design and Testing (SatMDT) [15] was applied to the development of persuasive messages in this study. The SatMDT draws on well-known social psychological theories of persuasion, decision making, and attitude-behavior relations, and proposes the four-step process for devising key aspects of message content likely to enhance message persuasiveness. The step 1 (pre-existing individual characteristics) and the step 2 (message-related characteristics) relate to developing message concepts, deciding what messages to develop, and developing the messages. The step 3 (individual responses) and the step 4 (message outcomes) relate to pretesting the messages and assessing their effectiveness. Through the four-step process, users can complete the actions to be undertaken when they develop concepts, messages, and materials [5].

The study protocol was approved by the ethics committees of the Jikei University School of Medicine (reference number 28-223(8466)) and has been conducted in accordance with the Ethical Guidelines for Medical and Health Research Involving Human Subjects by the Japanese Government.

\section{Messages}

In accordance with the step 1 (pre-existing individual characteristics) and the step 2 (message-related characteristics), we developed three prototypes of persuasive messages. The aim of messaging was to promote help-seeking intentions for depression by imparting knowledge about depression. The target audience was the general public, excluding individuals who had received medical treatment for their mental disorders. As a general rule in Japan, people who report depressive symptoms such as depressed mood and disturbed sleep for over 2 weeks often screen positive for suspected depression, who are encouraged to seek advice and assistance from health professionals. The persuasive messages were designed to incorporate this approach. 
The persuasive messages used in this study were shown in Additional file 1. Each message consisted of three parts. The first part was the primary message statement. The second part provided information regarding the early signs of depression, that is "Depression can be recognized early by mental symptoms such as depressed mood, loss of interest, etc. and physical symptoms such as disturbed sleep, increased fatigue, etc.". The last part was a call to action, that is "if you think you might be depressed, don't worry alone and speak with your familiar primary care doctor.".

The three primary message statements were selected from the list of text messages devised by Bell and colleagues [16] so as to be matched with the beliefs related to the top three following reasons, respectively, for having no help-seeking intentions for depression, respectively [17]: (1) depression can happen to anyone, (2) depression needs treatment, and (3) depression improves with treatment. The first one was neutral framed with additional information on incidence of depression: about one out of 15 people experience depression during their lifetime. The second one was loss framed (thereat appeal) with additional information on prognosis of untreated patients: about $80 \%$ of untreated patients will not recover. The third one was gain framed (benefit appeal) with additional information on prognosis of treated patients: about $80 \%$ of treated patients will recover.

Formatted and unformatted versions of the three differently framed messages containing the identical sentences were prepared. The formatted versions were designed in accordance with the CDC Clear Communication Index User Guide [18], while the unformatted versions were designed in plain text. No significant differences in participants' assessment (comprehensibility and persuasiveness) were observed between the formatted and unformatted versions [14]. Therefore, the responses were lumped together in this study.

\section{Participants}

In accordance with the step 3 (individual responses) and the step 4 (message outcomes), we pretested the messages and assessed their effectiveness. A web-based survey was conducted in July 2017 on Japanese adults aged 35-45 years [14]. All participants were recruited from an online research panel of a leading research company in Japan (Cross Marketing Inc., Tokyo, Japan). Recruitment emails were sent to 8241 randomly selected eligible registrants, with medical professionals being excluded through a prescreening process. Potential participants in the present survey were accepted in the order of receipt until the quotas were met for gender, area, and psychological distress level as measured by the Japanese version of the 6-item Kessler Psychological Distress Scale (K6) [19]. A validation study revealed that the optimal cut-off point for the Japanese version of the $\mathrm{K} 6$ was estimated at $4 / 5$ with sensitivity of $100 \%$ and specificity of $68.7 \%$ [20]. The participants were classified as either distressed (K6 score $\geq 5)$ or non-distressed (K6 score $<5$ ) according to their $\mathrm{K} 6$ scores.

A total of 2520 responses were received over 2 days of recruitment. All participants agreed to participate in the survey voluntarily after reading a description of the purpose and procedures. Completion and submission of the survey was taken to indicate consent to participate. Of the 2520 respondents, 563 had received medical treatment for their mental disorders and therefore excluded. The remaining 1957 participants were grouped into the distressed (K6 score $\geq 5, N=824$ ) and non-distressed (K6 score $<5, N=1133$ ) groups and analyzed.

\section{Measures}

The participants randomly received one of three persuasive messages that aimed to promote help-seeking intentions for depression, and after reading the message for at least $15 \mathrm{~s}$, asked to rate it in terms of comprehensibility, persuasiveness, emotional response, and intended future use. Help-seeking intention for depression was measured using vignette methodology before and after exposure to the messages. The online questionnaire forms presented the questions one after one through the operation of a "Next" button. Respondents answered one question per page and were unable to go back to the previous page.

\section{Comprehensibility}

Five items on the perceived effectiveness rating scales [13] were used to ask how easy or difficult the information was to (1) read, (2) understand, (3) remember, (4) locate important information, and (5) keep for future reference. The scores for all items (range 1-5 points) were averaged to produce the comprehensibility score.

\section{Persuasiveness}

Seven items on the perceived effectiveness rating scales [13] were used to ask to what extent they agreed or disagreed that the information (1) was believable, (2) was convincing, (3) was important to me, (4) helped me feel confident about how best to do, (5) would help my family and friends, (6) put thoughts in my mind about wanting to do something, and (7) was agreeable. The scores for all items (range 1-5 points) were averaged to produce the persuasiveness score.

\section{Emotional response}

Participants were asked to what extent the message made them feel (1) surprised, (2) angry, (3) fearful, (4) sad, (5) guilty, (6) anxious, and (7) happy. The response options were from 1 (not at all) to 5 (extremely) [21, 22]. 


\section{Intended future use}

Future use of the message was measured by asking participants, "If you saw the information in a newspaper or magazine, how likely would you [use, read, and keep] it?" [17]. Participants responded to this question on a five-point scale ranging from 1 (very unlikely) to 5 (very likely).

\section{Help-seeking intention for depression}

Vignette methodology was used to measure help-seeking intention $[13,14,17]$. Participants were presented with a vignette describing a man (or woman) with depression and then asked "If you had health problems right now like Mr. A (or Ms. A), would you seek professional help?". The vignette was devised to represent two core symptoms (depressed mood and loss of interest) and one associated symptom (insomnia) of major depressive disorders based on the fifth edition of the Diagnostic and Statistical Manual of Mental Disorders (DSM). A description of the vignette is as follows.

- Mr. A (Ms. A) is a 45-year-old office worker. Over the last 2 weeks, despite not having any trouble, he (she) has always been so depressed that he (she) has not felt like doing anything. He (She) has woken up frequently during the night and has not had enough sleep. Accordingly, he (she) cannot go about his (her) work. He (She) is not currently undergoing treatment for any diseases.

Participants responded to this question on a four-point scale (certainly yes/probably yes/probably not/certainly not). Those who provided affirmative answers (certainly yes and probably yes) were considered to have a positive help-seeking intention.

\section{Statistical analysis}

All statistical analyses were performed using SAS version 9.4 (SAS Institute, Cary, NC, USA). The $t$ test and $\chi_{2}$ test were used to compare characteristics of the distressed and non-distressed groups. The analysis of covariance with adjustment for sociodemographic characteristics (gender, education, marital status, and occupation) were used to assess the main and interaction effects of message and psychological distress. Proportion of variance explained $\left(\eta^{2}\right)$ was used to estimate the effect sizes; this is usually classified as small (0.01), medium (0.06), or large (0.14). The McNemar test was used to compare the proportions of participants who had reported having a positive help-seeking intention before and after exposure to the messages. Among participants having no help-seeking intention before exposure to the messages, multiple logistic regression analysis was performed to examine the factors associated with increased help-seeking intention for depression with adjustment for sociodemographic characteristics (gender, education, marital status, and occupation). Adjusted odds ratios (ORs) with 95\% confidence intervals (CIs) for help-seeking intention for depression were calculated separately in the depressed and non-depressed groups. Significant levels were set at $p$ $<0.05$. In the assessment of main and interaction effects of message and psychological distress, Bonferroni adjustment was conducted for each test separately to consider the number of significance tests undertaken.

\section{Results}

Of the 824 participants of the distressed group, those who asked to rate messages 1,2 , and 3 were 270 (32.8\%), 267 (32.4\%), and 287 (34.8\%), respectively. Of the 1133 participants of the non-distressed group, these numbers were 382 (33.7\%), 382 (33.7\%), and 369 (32.6\%), respectively. Table 1 shows the characteristics of the study participants. No significant differences in sociodemographic characteristics were observed between the message groups in the distressed or non-distressed groups. Despite the random allocation, significant differences were observed between the distressed and non-distressed groups in most sociodemographic characteristics, as the distressed group was significantly more likely to be younger, have lower levels of educational attainment, be unmarried, have no occupation, and have lower income than the non-distressed group.

Table 2 shows the assessment of the persuasive messages. No significant differences $(p<0.05)$ in the comprehensibility and persuasiveness scores were observed between the messages, but the distressed group had significantly lower scores than the non-distressed group. Among the seven emotional responses, significant differences $(p<0.007)$ between the messages were found in fear, happiness, sadness, and anxiety, and significant differences between the distressed and non-distressed groups were found in all items except surprise. The highest scores for negative emotion items such as surprise, anger, fear, sadness, guilt, and anxiety were observed in message 2, while the highest score for a positive emotion item such as happiness was observed in message 3. Among the three items for intended future use, no significant differences $(p<0.016)$ were observed between the message, but the distressed group had a significantly higher score than the non-distressed group for keeping the message. The effect size estimates $\left(\eta^{2}\right)$ indicated that the effects were statistically significant but small. There were no significant interaction effects between message and psychological distress.

Table 3 shows the changes in help-seeking intention for depression before and after exposure to the messages. The proportion of participants who reported having a positive help-seeking intention for depression increased by $31.3 \%$ on average (changed from 30.2 to 
Table 1 Characteristics of the study participants

\begin{tabular}{|c|c|c|c|c|c|c|}
\hline & & \multicolumn{2}{|c|}{ Non-distressed } & \multicolumn{2}{|c|}{ Distressed } & $p$ \\
\hline \multicolumn{2}{|l|}{ N } & \multicolumn{2}{|l|}{1133} & \multicolumn{2}{|l|}{824} & \\
\hline k6 score & Mean (SD) & \multicolumn{2}{|c|}{$1.1(1.4)$} & \multicolumn{2}{|c|}{$10.3(4.4)$} & $<0.001$ \\
\hline Age & Mean (SD) & \multicolumn{2}{|c|}{$41.0(3.0)$} & \multicolumn{2}{|c|}{$40.7(3.0)$} & 0.012 \\
\hline \multirow[t]{2}{*}{ Gender } & Male & 570 & $50.3 \%$ & 410 & $49.8 \%$ & \multirow[t]{2}{*}{0.810} \\
\hline & Female & 563 & $49.7 \%$ & 414 & $50.2 \%$ & \\
\hline \multirow[t]{3}{*}{ Education } & Compulsory education/high school & 288 & $25.4 \%$ & 252 & $30.6 \%$ & \multirow[t]{3}{*}{0.032} \\
\hline & Junior college/vocational school & 306 & $27.0 \%$ & 218 & $26.5 \%$ & \\
\hline & University or higher & 539 & $47.6 \%$ & 354 & $43.0 \%$ & \\
\hline \multirow[t]{3}{*}{ Marital status } & Married & 698 & $61.6 \%$ & 403 & $48.9 \%$ & \multirow[t]{3}{*}{$<0.001$} \\
\hline & Unmarried & 381 & $33.6 \%$ & 386 & $46.8 \%$ & \\
\hline & Divorced/widowed & 54 & $4.8 \%$ & 35 & $4.2 \%$ & \\
\hline \multirow[t]{3}{*}{ Occupation } & Full-time job & 707 & $62.4 \%$ & 469 & $56.9 \%$ & \multirow[t]{3}{*}{0.049} \\
\hline & Temporary or part-time job & 181 & $16.0 \%$ & 148 & $18.0 \%$ & \\
\hline & No occupation & 245 & $21.6 \%$ & 207 & $25.1 \%$ & \\
\hline \multirow[t]{6}{*}{ Household income } & $<2.0$ million yen ${ }^{a}$ & 101 & $8.9 \%$ & 129 & $15.7 \%$ & \multirow[t]{6}{*}{$<0.001$} \\
\hline & 2.0-3.9 million & 196 & $17.3 \%$ & 198 & $24.0 \%$ & \\
\hline & 4.0-5.9 million & 333 & $29.4 \%$ & 219 & $26.6 \%$ & \\
\hline & 6.0-7.9 million & 246 & $21.7 \%$ & 154 & $18.7 \%$ & \\
\hline & 8.0-9.9 million & 140 & $12.4 \%$ & 65 & $7.9 \%$ & \\
\hline & $10.0+$ million & 108 & $9.5 \%$ & 53 & $6.4 \%$ & \\
\hline
\end{tabular}

${ }^{a} 1$ million yen was about 10,000 US dollars at the time of the survey

$39.7 \%$ ) in the distressed group and by $13.0 \%$ on average (changed from 42.1 to $47.6 \%$ ) in the non-distressed group. Significant increases in help-seeking intentions for depression were observed in all message groups both in the distressed and non-distressed groups.

In order to examine the factors associated with increased help-seeking intention for depression, multiple logistic regression analysis was conducted among 1231 participants (575 distressed and 656 non-distressed) having no help-seeking intention before exposure to the messages. When the three persuasive messages were compared (Table 4), message 2 had a significantly greater effect of increasing help-seeking intentions for depression in the non-distressed group. Meanwhile, no significant differences were observed between the messages in the distressed group. When the association between message assessment and increased help-seeking intention for depression was examined (Table 5), the persuasiveness score was significantly associated with increased help-seeking intention both in the distressed and non-distressed groups. In addition, the emotional responses of "surprise" and "guilt" had a significant association with increased help-seeking intention in the non-distressed and distressed groups, respectively.

\section{Discussion}

This study attempted to develop effective persuasive messages in accordance with the SatMDT to promote help-seeking intentions for depression. The study participants grouped into the distressed and non-distressed groups showed significant differences in the reactions to the messages (Table 2). Meanwhile, significant increases in help-seeking intentions for depression were observed after exposure to the messages both in the distressed and non-distressed groups (Table 3). Although this study did not provide enough evidence to deny the boomerang effect of depression help-seeking messages [9, 10], it should be noted that the exposure to persuasive messages produced good results to promote help-seeking intentions for depression irrespective of whether the audience were distressed or not.

The distressed group was significantly more likely to have lower levels of educational attainment, have no occupation, and have lower income than the non-distressed group (Table 1). Although this study did not clarify their causal relationships due to the cross-sectional design, the inverse association between socioeconomic status and mental health has been observed in many countries including Japan [23, 24]. We previously reported that people with low socioeconomic status were more likely to have poor health literacy skills, less likely to get sufficient health information, and in turn, more likely to have risky health habits and poor health status [25]. It may be important to design persuasive messages targeting people with depression to be easily understandable for people with poor health 
Table 2 Assessment of the persuasive messages encouraging help-seeking for depression

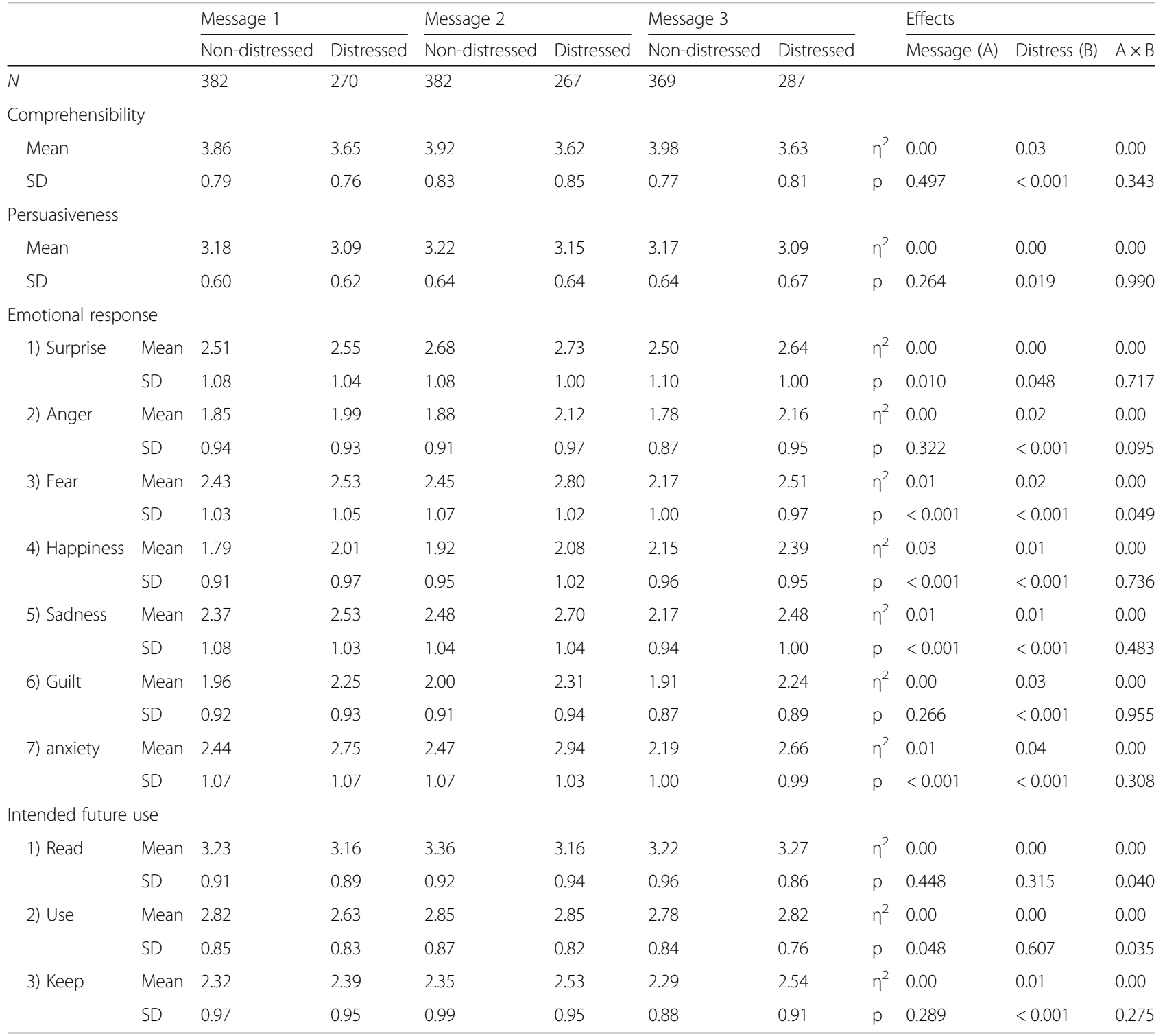

All items were scored on a 1 to 5 point scale. The effects of message (A), distress (B), and A $\times$ B were estimated with adjustment for gender, education, marital status, and occupation. Effect sizes were estimated by proportion of variance explained $\left(\eta^{2}\right)$, which is usually classified as small 0.01 , medium 0.06 , and large 0.14

Table 3 Changes in help-seeking intention for depression before and after exposure to the messages

\begin{tabular}{|c|c|c|c|c|c|c|c|c|c|c|}
\hline & \multicolumn{4}{|c|}{ Non-distressed } & \multirow[t]{3}{*}{$p$} & \multicolumn{4}{|c|}{ Distressed } & \multirow[t]{3}{*}{$p$} \\
\hline & \multirow[t]{2}{*}{$N$} & \multicolumn{3}{|c|}{ Positive intention } & & \multirow[t]{2}{*}{$N$} & \multicolumn{3}{|c|}{ Positive intention } & \\
\hline & & Before & After & Percentage change & & & Before & After & Percentage change & \\
\hline \multirow[t]{2}{*}{ Message 1} & 382 & 157 & 167 & & & 270 & 74 & 100 & & \\
\hline & & $41.1 \%$ & $43.7 \%$ & $+6.4 \%$ & 0.258 & & $27.4 \%$ & $37.0 \%$ & $+35.1 \%$ & $<0.001$ \\
\hline \multirow[t]{2}{*}{ Message 2} & 382 & 162 & 190 & & & 267 & 81 & 107 & & \\
\hline & & $42.4 \%$ & $49.7 \%$ & $+17.3 \%$ & 0.004 & & $30.3 \%$ & $40.1 \%$ & $+32.1 \%$ & $<0.001$ \\
\hline \multirow[t]{2}{*}{ Message 3} & 369 & 158 & 182 & & & 287 & 94 & 120 & & \\
\hline & & $42.8 \%$ & $49.3 \%$ & $+15.2 \%$ & 0.008 & & $32.8 \%$ & $41.8 \%$ & $+27.7 \%$ & $<0.001$ \\
\hline
\end{tabular}


Table 4 Effects of increasing help-seeking intentions for depression among participants having no help-seeking intention before exposure to the messages

\begin{tabular}{llllll}
\hline & \multicolumn{2}{l}{ Non-distressed } & & \multicolumn{2}{l}{ Distressed } \\
\cline { 2 - 3 } & OR & $95 \% \mathrm{Cl}$ & & OR & $95 \% \mathrm{Cl}$ \\
\hline Message 1 & 1.00 & (reference) & & 1.00 & (reference) \\
Message 2 & 1.67 & $(1.07-2.62)$ & 0.97 & $(0.58-1.60)$ \\
Message 3 & 1.48 & $(0.93-2.34)$ & & 0.90 & $(0.54-1.49)$ \\
\hline
\end{tabular}

Odds ratios (ORs) with $95 \%$ confidence intervals (Cls) were calculated with adjustment for gender, education, marital status, and occupation

literacy skills. Pretesting messages with audience members is also useful for checking the understandability of the wording of the messages.

The distressed group rated the persuasiveness of messages significantly lower than non-distressed group. Meanwhile, the emotional responses to the messages were more prominent in the distressed group, and the distressed group showed greater increases in their help-seeking intentions for depression after exposure to the messages than the non-distressed group. People with mental illness tend to have a negative attitude and low intention to seek professional help [9, 26, 27], as indeed the proportion of participants who reported having a positive help-seeking intention for depression before exposure to the messages was significantly lower in the distressed group (30.2\%) than in the non-distressed group (42.1\%). However, the results of this study suggested that individuals with depression may be more susceptible to the persuasive messages encouraging professional help seeking for depression. Most individuals with depression may be afraid that they need to seek medical treatment for their present depressive status, and thus they are more likely to receive the messages as

Table 5 Association between message assessment and increased help-seeking intention for depression among participants having no help-seeking intention before exposure to the messages

\begin{tabular}{llllll}
\hline & \multicolumn{2}{l}{ Non-distressed } & & \multicolumn{2}{l}{ Distressed } \\
\cline { 2 - 3 } & OR & $95 \% \mathrm{Cl}$ & & OR & $95 \% \mathrm{Cl}$ \\
\hline Comprehensibility & 1.16 & $(0.88-1.51)$ & & 1.38 & $(0.99-1.91)$ \\
Persuasiveness & 1.59 & $(1.10-2.30)$ & 1.77 & $(1.17-2.68)$ \\
Emotional responses & & & & \\
1) Surprise & 1.27 & $(1.04-1.55)$ & 1.07 & $(0.83-1.37)$ \\
2) Anger & 0.91 & $(0.66-1.23)$ & 0.87 & $(0.60-1.26)$ \\
3) Fear & 1.13 & $(0.84-1.52)$ & 0.99 & $(0.70-1.39)$ \\
4) Happiness & 0.84 & $(0.65-1.08)$ & 1.23 & $(0.94-1.61)$ \\
5) Sadness & 1.10 & $(0.83-1.46)$ & 0.75 & $(0.52-1.08)$ \\
6) Guilt & 1.30 & $(0.91-1.85)$ & 1.62 & $(1.04-2.51)$ \\
7) Anxiety & 0.79 & $(0.60-1.05)$ & 0.84 & $(0.61-1.15)$ \\
\hline
\end{tabular}

Odds ratios (ORs) with 95\% confidence intervals (Cls) were calculated with adjustment for gender, education, marital status, and occupation self-relevant information. It seems reasonable that individuals with depression feel strongly sad when they are informed the loss-framed message, while they feel strongly happy when they are informed the gain-framed message, and also that individuals with depression think to keep the message. Recently, some investigators focused on whether increased levels of depressive symptomatology were associated with reduced help-seeking intentions $[28,29]$. Since the study participants consisted of individuals with no or mild depressive symptoms, further studies may be needed to confirm the susceptibility to the messages for individuals with severe depressive symptoms.

The multiple logistic regression analysis showed that message 2 (loss-framed; negative, threat appeal) had a significantly greater effect of increasing help-seeking intentions for depression in the non-distressed group. In the World Mental Health Japan Survey, the most frequently reported reason for not seeking treatment for their mental illness was a low perceived need (63.9\%) and that for delaying access to help for their mental illness was a desire to handle the problem on one's own (68.8\%) [30]. Message 2 seems to attack the major barrier of help-seeking depression directly as it was designed to emphasize the necessity of treatment. Even though no significant differences were observed between the messages in the distressed group, message 2 induced a comparative increase $(32.1 \%)$ in help-seeking intentions for depression. Therefore, compared with messages 1 and 3, message 2 may be suitable for public health interventions targeting all people irrespective of whether they were depressed or not distressed. This type of public health intervention would also be likely to alter public attitudes regarding depression, and disrupt the public stigma-induced negative feedback, thereby facilitating help-seeking [31].

The results of the present study provide evidence regarding the effectiveness of depression help-seeking messages. However, the study did have potential limitations. First, the web-based survey was self-administered, so the accuracy of the responses depends on the participants' understanding of the questions and motivation to answer them accurately. For example, the scores for the intended future use questions, which were derived from the Consumer Information Rating Form and originally applied to paper-based health information [13], were lower than expected; some of the participants may have found it difficult to imagine using or keeping the given message they saw on the screen, even though the wording of the items was checked for understandability prior to the survey. Using the Internet guaranteeing anonymity could be expected to elicit more truthful responses, through minimizing pressure in terms of social desirability [32]. However, it is nearly impossible to eliminate the 
risk of information bias completely. Second, the study participants were selected from a nationwide panel provided by a research company. According to a national census [33], the percentage of the Japanese population between the ages of 35 and 44 years with a university degrees was $22.0 \%$ in 2010; this number is considerably lower than in the present study (45.6\%). Therefore, the results may have been influenced to some extent by a selection bias. Third, the ages of the study participants were limited to 35-45 years. The Comprehensive Survey of Living Conditions conducted by the Japanese Ministry of Health, Labour, and Welfare revealed that individuals feeling stressed or distressed are most frequently observed in the $40-49$-year-old age group $58.7 \%$ in men and $48.6 \%$ in women) [34]. In addition, the World Mental Health Japan Survey revealed that the 12-month prevalence of mental disorders was significantly higher in the younger age groups [35]. Therefore, people aged 35-45 years seemed to be suitable target for persuasive messages encouraging professional help seeking for depression. The results of the present study supports the effectiveness of depression help-seeking messages; however, it is uncertain whether the messages will work equally well in other age groups. Fourth, this study used a pretest-posttest design to assess changes in help-seeking intention for depression. In order to prevent demand characteristics, participants asked to answer a series of questions impertinent to depression between the pre and post assessments. This distractor task may have reduced the occurrence of demand characteristics to some extent; however, the effect of messaging on help-seeking intention for depression shown in this study is likely to be overestimated. Fifth, this study could not determine the causal relationships due to the cross-sectional design. Intention is recognized as a key predictor of behavior, but the strength of the intention-behavior relationship can vary depending on the type of behavior [36]. There was no knowing whether the self-reported help-seeking intentions accurately reflect the actual help-seeking behaviors if they become mentally ill. Further studies are needed to determine whether the persuasive messages developed in this study can increase actual help-seeking behaviors.

\section{Conclusion}

The exposure to persuasive messages may promote help-seeking intentions for depression. It seems likely that loss framing will work better than neutral and gain framing. Meanwhile, the responses to persuasive messages may differ to some extent between distressed and non-distressed individuals, as individuals with psychological distress are likely to be more susceptible to persuasive messages than those without.

\section{Additional file}

Additional file 1: Persuasive messages encouraging help-seeking for depression. (DOC 280 kb)

\section{Abbreviations}

K6: 6-item Kessler Psychological Distress Scale; SatMDT: Step approach to Message Design and Testing

\section{Acknowledgements}

Not applicable.

\section{Funding}

This work was supported by the JSPS KAKENHI Grant Number 16 K09147 and the Uehara Memorial Foundation Research Grant.

\section{Availability of data and materials \\ Not applicable.}

\section{Authors' contributions}

MS was responsible for the design and conduct of the study, the collection, analysis, and interpretation of data, and the writing of the article. TY and HY contributed to the data interpretation and discussion of the implications of this work. All authors read and approved the final manuscript.

\section{Ethics approval and consent to participate}

The study protocol was approved by the ethics committee of the Jikei University School of Medicine (reference number 28-223(8466)) and has been conducted in accordance with the Ethical Guidelines for Medical and Health Research Involving Human Subjects by the Japanese Government. Consent to participate was implied by the completion and submission of the survey. Written informed consent was omitted with the approval from the ethics committee of the Jikei University School of Medicine.

\section{Consent for publication}

Not applicable.

\section{Competing interests}

The authors declare that they have no competing interests.

\section{Publisher's Note}

Springer Nature remains neutral with regard to jurisdictional claims in published maps and institutional affiliations.

Received: 24 January 2019 Accepted: 15 April 2019

Published online: 08 May 2019

\section{References}

1. Halfin A. Depression: the benefits of early and appropriate treatment. Am J Manag Care. 2007;13(4 Suppl):S92-7.

2. Wang PS, Angermeyer M, Borges G, Bruffaerts R, Tat Chiu W, DE Girolamo G, Fayyad J, Gureje O, Haro JM, Huang Y, Kessler RC, Kovess V, Levinson D, Nakane Y, Oakley Brown MA, Ormel JH, Posada-Villa J, Aguilar-Gaxiola S, Alonso J, Lee S, Heeringa S, Pennell BE, Chatterji S, Ustün TB. Delay and failure in treatment seeking after first onset of mental disorders in the World Health Organization's world mental health survey initiative. World Psychiatry. 2007;6:177-85.

3. Wang PS, Aguilar-Gaxiola S, Alonso J, Angermeyer MC, Borges G, Bromet EJ, Bruffaerts R, de Girolamo G, de Graaf R, Gureje O, Haro JM, Karam EG, Kessler RC, Kovess V, Lane MC, Lee S, Levinson D, Ono Y, Petukhova M, Posada-Villa J, Seedat S, Wells JE. Use of mental health services for anxiety, mood, and substance disorders in 17 countries in the WHO world mental health surveys. Lancet. 2007;370:841-50.

4. Gulliver A, Griffiths KM, Christensen H, Brewer JL. A systematic review of help-seeking interventions for depression, anxiety and general psychological distress. BMC Psychiatry. 2012;12:81.

5. National Cancer Institute. Making health communication programs work (Pink Book). Available at: https://www.cancer.gov/publications/healthcommunication (Accessed 15 July 2017). 
6. Akl EA, Oxman AD, Herrin J, Vist GE, Terrenato I, Sperati F, Costiniuk C, Blank $D$, Schünemann $H$. Framing of health information messages. Cochrane Database Syst Rev. 2011;12:CD006777.

7. Gallagher KM, Updegraff JA. Health message framing effects on attitudes, intentions, and behavior: a meta-analytic review. Ann Behav Med. 2012;43: 101-16.

8. Covey J. The role of dispositional factors in moderating message framing effects. Health Psychol. 2014;33:52-65.

9. Lienemann BA, Siegel JT, Crano WD. Persuading people with depression to seek help: respect the boomerang. Health Commun. 2013;28:718-28.

10. Niederkrotenthaler T, Reidenberg DJ, Till B, Gould MS. Increasing helpseeking and referrals for individuals at risk for suicide by decreasing stigma: the role of mass media. Am J Prev Med. 2014;47(3 Suppl 2):S235-43.

11. Bernhardt JM. Communication at the core of effective public health. Am J Public Health. 2004:94:2051-3.

12. Daniel $\mathrm{KL}$, Bernhardt JM, Eroğlu D. Social marketing and health communication: from people to places. Am J Public Health. 2009:99:2120-2.

13. Suka M, Yamauchi T, Yanagisawa H. Perceived effectiveness rating scales applied to insomnia help-seeking messages for middle-aged Japanese people: a validity and reliability study. Environ Health Prev Med. 2017;22:69.

14. Suka M, Yamauchi T, Yanagisawa H. Comparing responses to differently framed and formatted persuasive messages to encourage help-seeking for depression in Japanese adults: a cross-sectional study with 2-month followup. BMJ Open. 2018;8:e020823.

15. Lewis I, Watson B, White KM. The step approach to message design and testing (SatMDT): a conceptual framework to guide the development and evaluation of persuasive health messages. Accid Anal Prev. 2016;97:309-14.

16. Bell RA, Paterniti DA, Azari R, Duberstein PR, Epstein RM, Rochlen AB, Johnson MD, Orrange SE, Slee C, Kravitz RL. Encouraging patients with depressive symptoms to seek care: a mixed methods approach to message development. Patient Educ Couns. 2010;78:198-205.

17. Suka M, Yamauchi T, Sugimori H. Help-seeking intentions for early signs of mental illness and their associated factors: comparison across four kinds of health problems. BMC Public Health. 2016;16:301.

18. Centers for Disease Control and Prevention. CDC Clear Communication Index. Available at: http://www.cdc.gov/ccindex (Accessed 22 Apr 2018).

19. Kawakami N, Kondo K, Yanagida K, Furukawa T. Mental health research on the preventive measure against suicide in adulthood. In: Ueda S, editor. Report of the research grant for the implementation of preventive measure based on the current status of suicide from the Ministry of Health, Labour and Welfare. Tokyo: Ministry of Health, Labour and Welfare; 2005. p. 147-57. (in Japanese).

20. Sakurai K, Nishi A, Kondo K, Yanagida K, Kawakami N. Screening performance of K6/K10 and other screening instruments for mood and anxiety disorders in Japan. Psychiatry Clin Neurosci. 2011;65:434-41.

21. Dillard JP, Peck E. Affect and persuasion: emotional responses to public service announcements. Commun Res. 2000:27:461-95.

22. Dillard JP, Shen L, Vail RG. Does perceived message effectiveness cause persuasion or vice versa? 17 consistent answers. Hum Commun Res. 2007; 33:467-88

23. Sakurai K, Kawakami N, Yamaoka K, Ishikawa H, Hashimoto H. The impact of subjective and objective social status on psychological distress among men and women in Japan. Soc Sci Med. 2010;70:1832-9.

24. Honjo K, Kawakami N, Tsuchiya M, Sakurai K. Association of subjective and objective socioeconomic status with subjective mental health and mental disorders among Japanese men and women. Int J Behav Med. 2014;21:421-9.

25. Suka M, Odajima T, Okamoto M, Sumitani M, Igarashi A, Ishikawa H, Kusama M, Yamamoto M, Nakayama T, Sugimori H. Relationship between health literacy, health information access, health behavior, and health status in Japanese people. Patient Educ Couns. 2015;98:660-8.

26. Rüsch N, Angermeyer MC, Corrigan PW. Mental illness stigma: concepts, consequences, and initiatives to reduce stigma. Eur Psychiatry. 2005;20:529-39.

27. Gulliver A, Griffiths KM, Christensen H. Perceived barriers and facilitators to mental health help-seeking in young people: a systematic review. BMC Psychiatry. 2010;10:113

28. Lienemann BA, Siegel JT. State psychological reactance to depression public service announcements among people with varying levels of depressive symptomatology. Health Commun. 2016:31:102-16.

29. Lueck JA. Matching message design and depressed cognition: an exploration of attention patterns for gain- and loss-framed depression helpseeking messages. J Health Commun. 2017;22:593-603.
30. Kanehara A, Umeda M, Kawakami N. Barriers to mental health care in Japan: results from the world mental health Japan survey. Psychiatry Clin Neurosci. 2015;69:523-33

31. Evans-Lacko S, Brohan E, Mojtabai R, Thornicroft G. Association between public views of mental illness and self-stigma among individuals with mental illness in 14 European countries. Psychol Med. 2012:42:1741-52.

32. Joinson A. Social desirability, anonymity, and internet-based questionnaires. Behav Res Methods Instrum Comput. 1999;31:433-8.

33. Ministry of Internal Affairs and Communications. National Census (in Japanese). Available at: https://www.e-stat.go.jp/SG1/estat/GL02100104. do?tocd $=00200521$ (Accessed 17 Nov 2018).

34. Ministry of Health, Labour, and Welfare. Comprehensive Survey of Living Conditions 2016 (in Japanese). Available at: http://www.mhlw.go.jp/toukei/ saikin/hw/k-tyosa/k-tyosa16/ (Accessed 17 Nov 2018).

35. Ishikawa H, Kawakami N, Kessler RC. Lifetime and 12-month prevalence, severity and unmet need for treatment of common mental disorders in Japan: results from the final dataset of world mental health Japan survey. Epidemiol Psychiatr Sci. 2016;25:217-29.

36. Sheeran P. Intention-behavior relations: a conceptual and empirical review. Eur Rev Soc Psychol. 2002;12:1-36.

\section{Ready to submit your research? Choose BMC and benefit from:}

- fast, convenient online submission

- thorough peer review by experienced researchers in your field

- rapid publication on acceptance

- support for research data, including large and complex data types

- gold Open Access which fosters wider collaboration and increased citations

- maximum visibility for your research: over $100 \mathrm{M}$ website views per year

At BMC, research is always in progress.

Learn more biomedcentral.com/submissions 